\title{
Chronic Kidney Disease: Implications for Progression Using Telehealth Monitoring
}

Abeera Mansur, Rehan Javed

Background: Chronic Kidney Disease (CKD) is a complex and common medical condition requiring a multidisciplinary management approach, including control of multiple parameters to delay progression of the disease. Telehealth has been used for monitoring chronic conditions and can be used for managing chronic kidney disease. Materials and Methods: Patients $(N=34)$ with CKD Stage II-V were studied for four months. Vital signs, weight, intake and output, blood glucose level, oxygen saturation, and edema were checked twice weekly by trained nurses, transmitted via WhatsApp, and incorporated into the electronic medical records of the patients. Monthly serum creatinine, hemoglobin, and electrolytes were obtained and reviewed by a nephrologist. Necessary intervention was conveyed to the patients.

Results: Patient satisfaction rate with telehealth management was high. Measured parameters were maintained in the recommended range in a higher percentage of the study group compared to the controls. Glomerular filtration rate was maintained in a significantly more patient in the study group $(p<0.001)$.

Conclusions: Telehealth monitoring of patients with CKD (tele-nephrology) is a viable and effective option for management of this increasingly prevalent disease.

Chronic kidney disease (CKD) is a worldwide public health problem with increasing severity in renal dysfunction extending to dialysis.

One in 10 American adults has some level of CKD (Table 1) according to the National Institute of Diabetes and Digestive and Kidney Diseases (NIDDK). ${ }^{1}$ According to 19992004 NHANES data, the estimated prevalence of CKD by stage is as follows: Stage 1: 5.7\%; Stage 2: 5.4\%; Stage 3: 5.4\%; Stage 4: 0.4\%, Stage 5: 0.4\%. ${ }^{2}$

Table 1. Stages of chronic kidney disease. 


\begin{tabular}{|c|c|}
\hline Stage 1 & Kidney damage ${ }^{*}$ with normal or increased GFR $\left(>90 \mathrm{~mL} / \mathrm{min} / 1.73 \mathrm{~m}^{2}\right)$ \\
\hline Stage 2 & Mild reduction in GFR $\left(60-89 \mathrm{~mL} / \mathrm{min} / 1.73 \mathrm{~m}^{2}\right)$ \\
\hline Stage $3 a$ & Moderate reduction in GFR $\left(45-59 \mathrm{~mL} / \mathrm{min} / 1.73 \mathrm{~m}^{2}\right)$ \\
\hline Stage 3b & Moderate reduction in GFR $\left(30-44 \mathrm{~mL} / \mathrm{min} / 1.73 \mathrm{~m}^{2}\right)$ \\
\hline Stage 4 & Severe reduction in GFR $\left(15-29 \mathrm{~mL} / \mathrm{min} / 1.73 \mathrm{~m}^{2}\right)$ \\
\hline Stage 5 & Kidney failure (GFR $<15 \mathrm{~mL} / \mathrm{min} / 1.73^{2}$ or dialysis) \\
\hline
\end{tabular}

The prevalence of Stages 3 and 4 CKD increased in the U.S. between 1988 and 2002, stabilized from 2003 to 2012, and may now be rising again. ${ }^{3,4}$ The prevalence of CKD in adults 30 years or older is projected to increase to $16.7 \%$ in 2030 , and for an individual, lifetime risk of CKD is high, with more than half the US adults aged 30 to 64 years likely to develop CKD. Worldwide, an estimated global CKD prevalence of $11 \%$ to $13 \%$ was reported in a systematic review and meta-analysis of observational studies. ${ }^{8}$

CKD management requires a systematic approach that can benefit from telemedicine. The objective of this study was to apply telehealth to optimize care of CKD and ensure control of parameters known to slow progression of the disease. Electronic submission was utilized to ensure intensive monitoring and intervention.

\section{Materials and Methods}

A total of 74 patients were recruited for the study. Patients $(n=40)$ with documented CKD Stages2-4 were enrolled for home visits by trained nurses. Patients with known CKD Stages II-V were included. Table 2 shows demographic and clinical characteristics.

Table 2: Demographic and characteristics of patients. 


\begin{tabular}{|l|c|c|}
\hline & Study & Control \\
\hline Age (Mean; yr) \pm SD & $65.6 \pm 13.1$ & $65.8 \pm 9.4$ \\
\hline Male/Female & $10 / 24$ & $18 / 15$ \\
\hline CKD II & 2 & 2 \\
\hline CKD III & 11 & 11 \\
\hline CKD IV & 15 & 15 \\
\hline CKD V & 6 & 6 \\
\hline DM & 24 & 25 \\
\hline HTN & 33 & 33 \\
\hline IHD & 19 & 17 \\
\hline
\end{tabular}

CKD: chronic kidney disease; DM: diabetes mellitus; HTN: hypertension; IHD; Ischemic Heart Disease

Patients on hemodialysis or who had renal transplant were excluded, as were patients less than 18 years-old. Consent was obtained for monitoring and transmission of parameters. Parameters were checked and transmitted via WhatsApp twice weekly, including temperature, blood pressure, pulse, respiratory rate, weight, intake and output, edema, oxygen saturation and serum glucose levels. These parameters were entered into the patient's electronic medical record and reviewed daily.

Temperature greater than $38^{\circ} \mathrm{C}\left(100.4^{\circ} \mathrm{F}\right)$, heart rate less than 60 beats per minute and more than 100 beats per minute, blood pressure higher than $140 / 90 \mathrm{mmHg}$ or lower than $100 / 60 \mathrm{mmHg}$, weight gain, uncontrolled serum glucose levels, edema, decreased cardiac output, and decreased oxygen saturation were identified.

Data transmission and intervention were done by the physician. Monthly hemoglobin, serum creatinine/GFR (glomerular filtration rate) and electrolytes (serum potassium and bicarbonate) were obtained and transmitted to the physician. Medications were adjusted accordingly. Medication, dietary and vaccination compliance, avoidance of nephrotoxic 
drugs, cessation of smoking, and CKD education were ensured. Home monitoring of patients was done over four months.

Similarly, control patients $(n=34)$ with CKD Stages II-V were identified. These patients had monthly physician office visits.

Baseline medical records were available for both groups, including medical history and physical, medications, diagnosis, and previous laboratory results. At the end of the study, patients were surveyed to determine satisfaction.

\section{Results}

At the end of the study there were 34 patients in each arm due to non-compliance and one deceased patient secondary to cardiac arrest.

A combination of the Service User Technology Acceptability Questionnaire (SUTAQ) and the 10-item Telehealth Satisfaction Scale Patient Satisfaction survey was used at the end of the study. Questions were modified according to the study design. Interviews were conducted by a non-participating individual. Ten "Yes/No" format questions were asked of patients in the study group.

The survey revealed that $91 \%$ of patients wanted to continue with the telemedicine service, $66 \%$ thought it was cost effective.

Only $12 \%$ had to visit an emergency department, while clinic visits decreased in $73 \%$ of patients. Emergency support available through this service was satisfactory for $61 \%$; $91 \%$ were taught the importance of monitoring labs, complying with medication, vaccine and diet (Figure 1).

In $89 \%$ there was improved compliance with the prescribed diet and medications. Most patients believed that confidentiality was maintained; $84 \%$ were happy with the clinical management and $91 \%$ with the nursing care (Figure 2). 


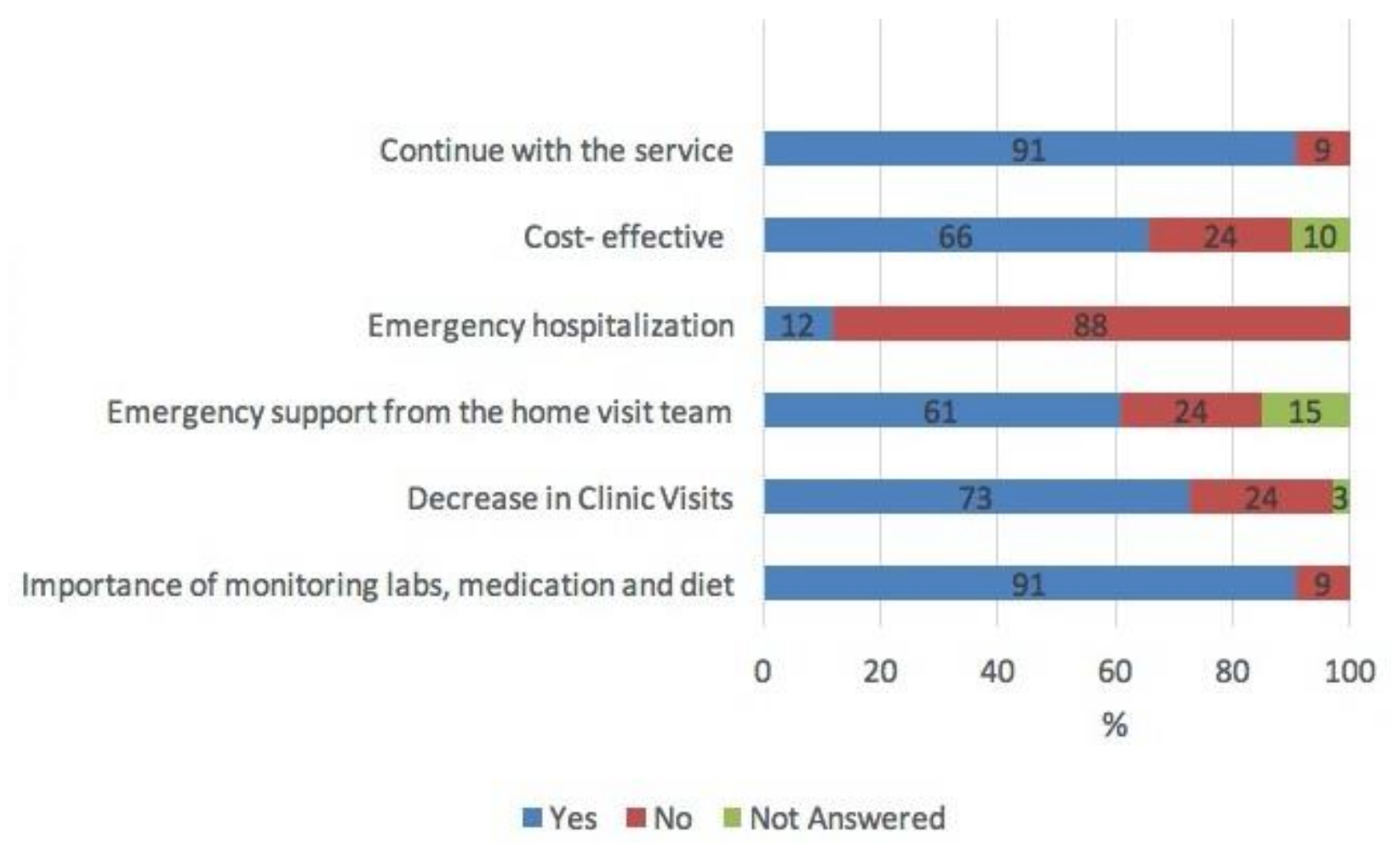

Figure1. Patient responses to telemedicine. 


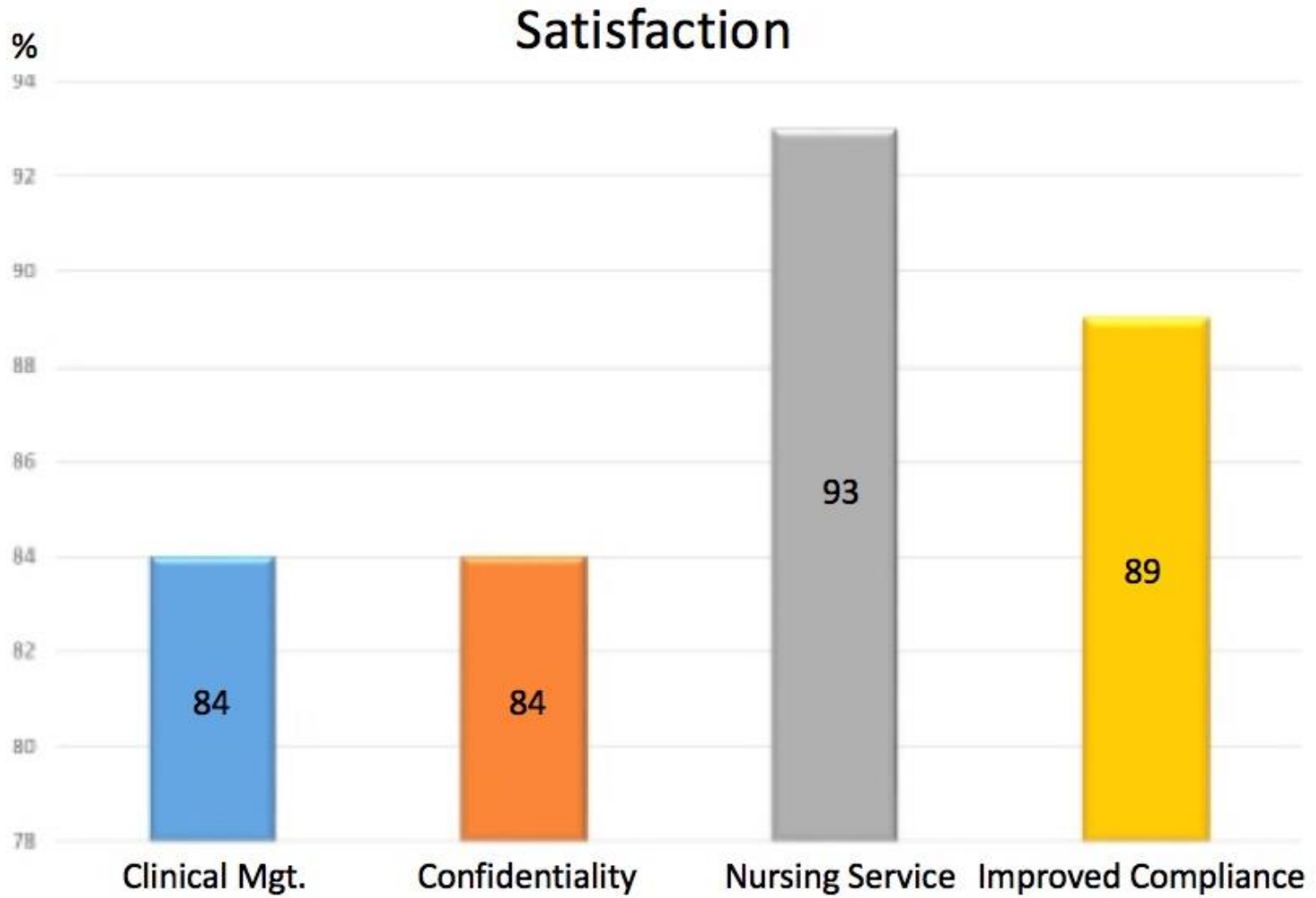

Figure 2. Satisfaction with telemedicine service.

Statistical analysis of the clinical component of the study was done using Independent2-Sample $t$ test to compare means for two groups (Table 3). A p value of less than 0.05 was statistically significant. In the comparison of control versus study patients, blood pressure control $(p<0.001)$, weight control $(p<0.001)$, medication compliance $(p=0.009)$, and vaccination compliance $(p=0.002)$ were significantly better in the study group (Figure 3).

Table 3. Statistical analysis. 


\begin{tabular}{|c|c|c|c|c|c|c|c|c|}
\hline & Groups & $\mathrm{N}$ & Mean & SD & $\mathrm{F}$ & Sig. & $\mathrm{t}$ & df \\
\hline \multirow[t]{2}{*}{ BP control } & study & 34 & .9412 & .23883 & & & 2.365 & 66 \\
\hline & control & 34 & .7353 & .44781 & 29.701 & $<.0001$ & 2.365 & 50.368 \\
\hline \multirow[t]{2}{*}{ Weight control } & study & 34 & .8824 & .32703 & & & 2.345 & 66 \\
\hline & control & 34 & .6471 & .48507 & 25.457 & $<.0001$ & 2.345 & 57.863 \\
\hline \multirow{2}{*}{$\begin{array}{l}\text { Medication } \\
\text { Compliance }\end{array}$} & study & 34 & .7941 & .41043 & & & 1.350 & 66 \\
\hline & control & 34 & .6471 & .48507 & 7.279 & 0.009 & 1.350 & 64.239 \\
\hline \multirow[t]{2}{*}{ Vaccination } & study & 34 & .7941 & .41043 & & & 3.742 & 66 \\
\hline & control & 34 & .3824 & .48507 & 10.007 & 0.002 & 3.742 & 63.88 \\
\hline \multirow[t]{2}{*}{ HB Control } & study & 34 & .9118 & .28790 & & & 2.204 & 66 \\
\hline & control & 34 & .7059 & .46250 & 23.779 & $<.0001$ & 2.204 & 55.236 \\
\hline \multirow{2}{*}{$\begin{array}{l}\text { Glycemic } \\
\text { Control }\end{array}$} & study & 24 & .9167 & .28233 & & & 2.105 & 47 \\
\hline & control & 25 & .6800 & .47610 & 23.205 & $<.0001$ & 2.126 & 39.301 \\
\hline \multirow[t]{2}{*}{ Electrolyte } & study & 34 & 1.0000 & .00000 & & & 4.243 & 66 \\
\hline & control & 34 & .6471 & .48507 & 348.48 & $<.0001$ & 4.243 & 33.000 \\
\hline \multirow[t]{2}{*}{ Hospitalization } & study & 34 & .0294 & .17150 & & & -.583 & 66 \\
\hline & control & 34 & .0588 & .23883 & 1.388 & 0.243 & -.583 & 59.84 \\
\hline \multirow[t]{2}{*}{ Education } & study & 34 & 1.0000 & .00000 & & & 1.000 & 66 \\
\hline & control & 34 & .9706 & .17150 & 4.254 & 0.043 & 1.000 & 33.000 \\
\hline \multirow[t]{2}{*}{ GFR } & study & 34 & .9118 & .28790 & & & 4.415 & 66 \\
\hline & control & 34 & .4706 & .50664 & 67.770 & $<.0001$ & 4.415 & 52.300 \\
\hline
\end{tabular}

BP: blood pressure; df: degrees of freedom; F: F-test; GFR: glomerular filtration rate; $H B$ : hemoglobin; $S D$ : standard deviation; $t$ : $t$ distribution or the Student $t$ 


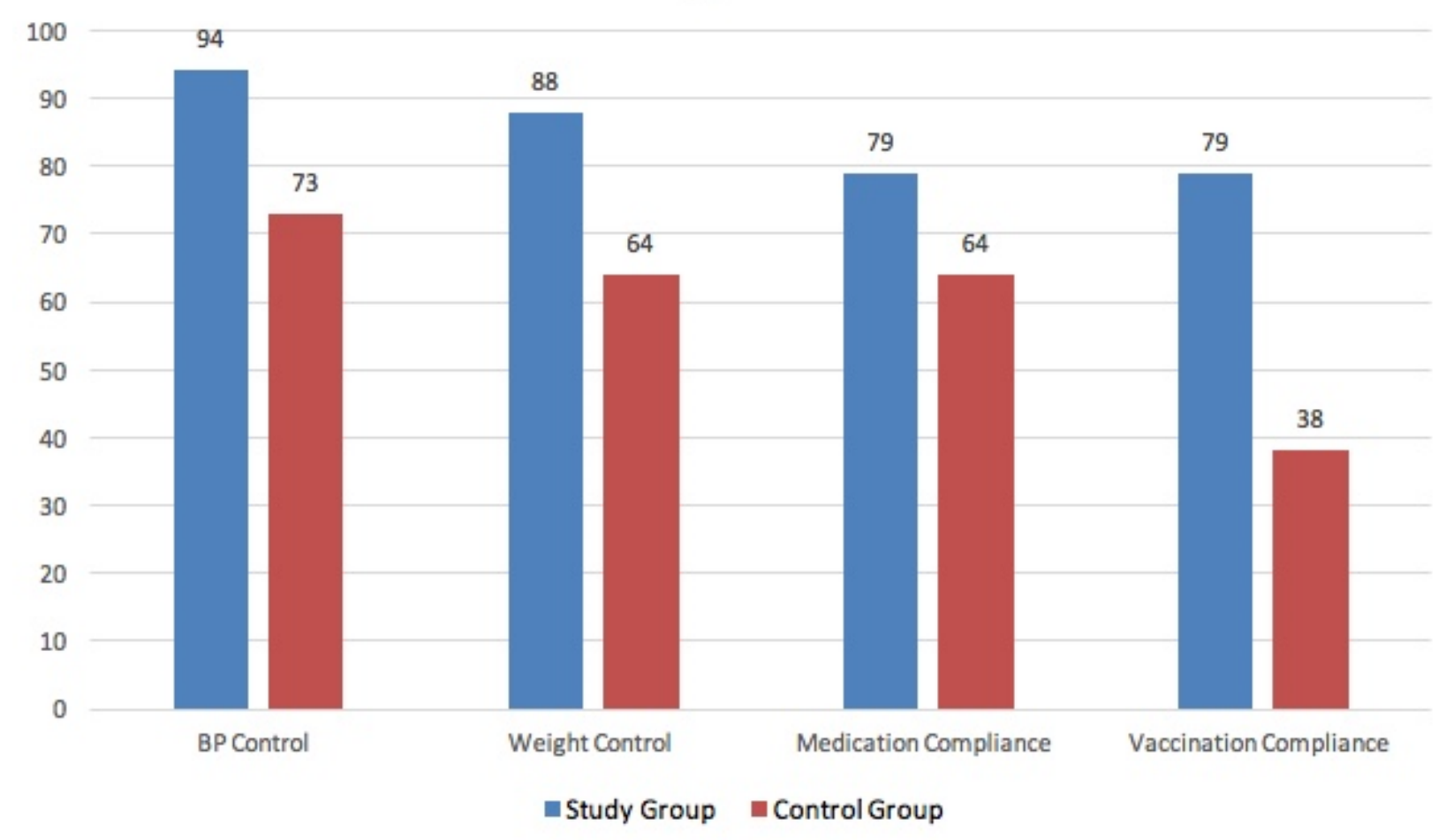

Figure 3. Comparison of blood pressure $(B P)$ and weight control, medication and vaccination compliance between study and control groups.

In the control population, $88 \%$ of patients were compliant with laboratory investigations compared to $100 \%$ of the study population. Hemoglobin at more than $11.5 \mathrm{~g} / \mathrm{dL}$ (target defined by KDIGO (Kidney Disease: Improving Global Outcome) was achieved in the study population $(p<0.001)$. Glycemic control was better and electrolytes were maintained within normal range in the study population. $(p<0.001)$.

Rate of hospitalization was not different (Figure 4). Both groups were educated about chronic kidney disease. GFR either improved or remained stable in a significantly larger percentage of the study population compared to the control population $(p \leq 0.001$; Figure $5)$. 


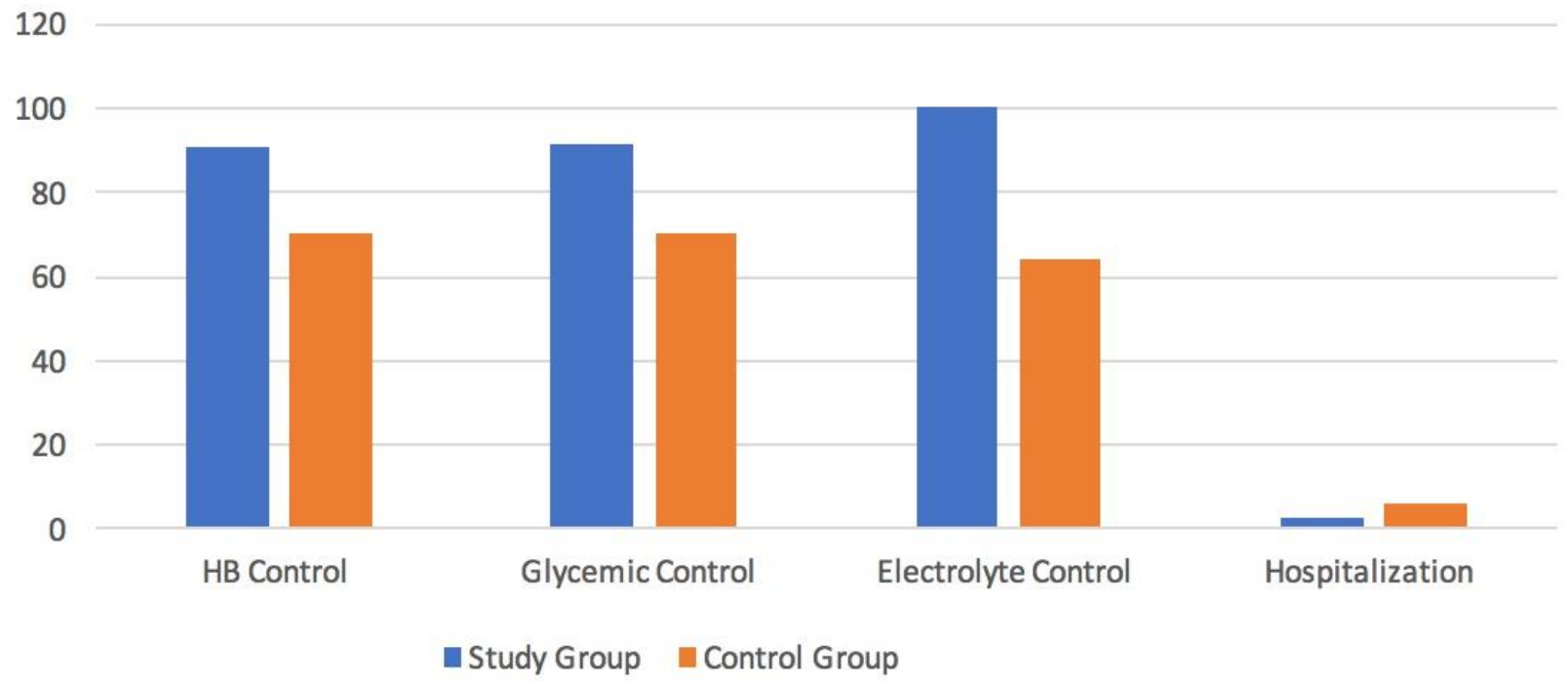

Figure 4. Comparison of hemoglobin (HB), glycemic, electrolyte control and hospitalizations between study and control groups.

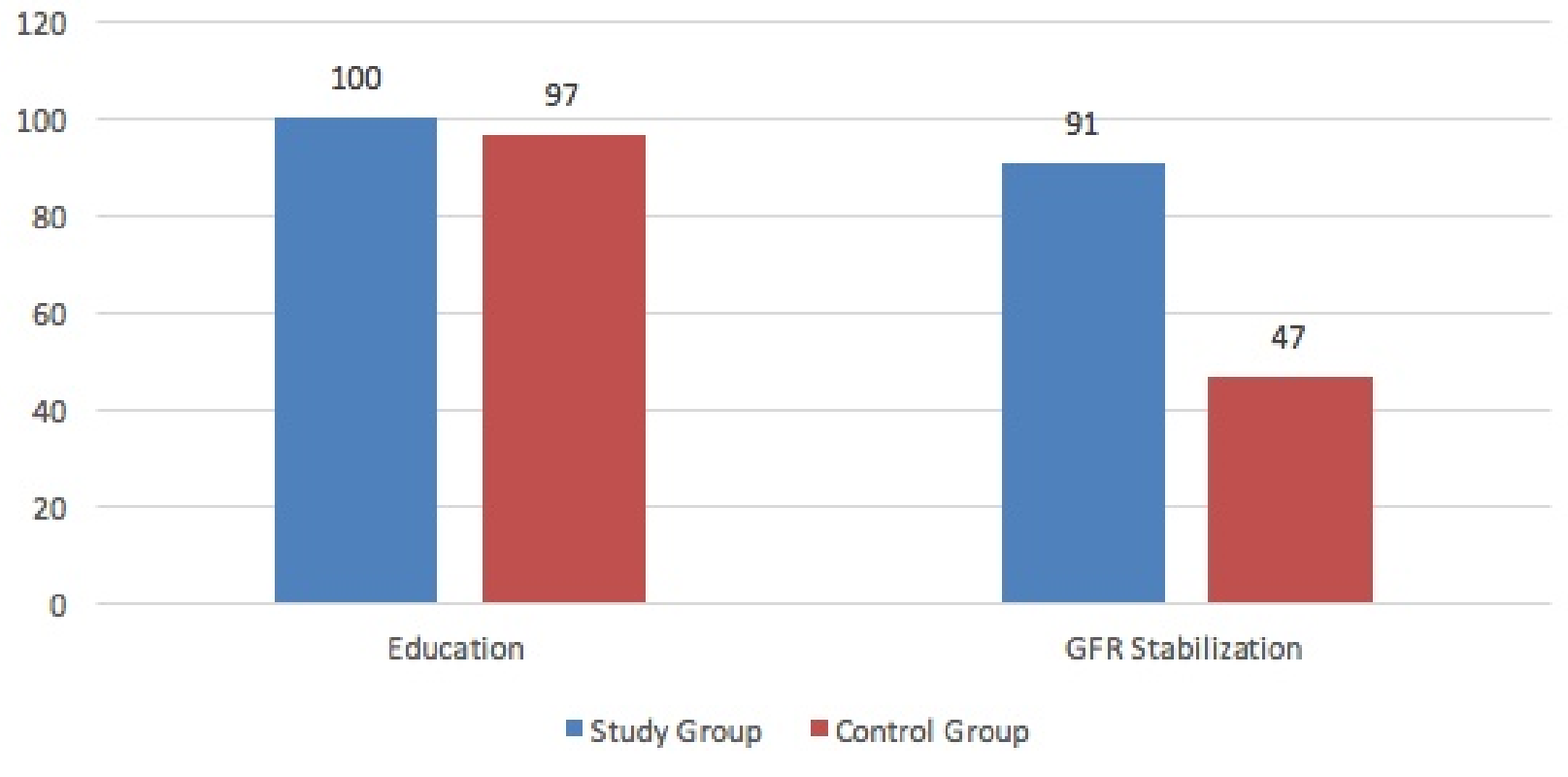

Figure 5. Comparison of education and glomerular filtration rate (GFR) stabilization between study and control groups.

\section{Discussion}


In this study, WhatsApp Messenger was used to transmit data. WhatsApp is a crossplatform, and end-to-end encrypted instant messaging application for smart phones. This freeware uses the Internet to make voice calls, one-to-one video calls; send text messages, images, GIF (graphics interchange format), videos, documents, user location, audio files, phone contacts, and voice notes to other users using standard cellular mobile numbers. I addition, it incorporates a feature called Status, which allows users to upload photos and videos to a 24-hours-lifetime feed that, by default, are visible to all contacts, similar to Snapchat, Facebook and Instagram Stories.

Patients with chronic kidney disease have multiple medical problems, including hypertension, diabetes, ischemic heart disease, and congestive heart failure. In addition, they have other conditions such as anemia, mineral and bone disease changes, and electrolyte abnormalities. Thus, they are complex to manage.

Mortality rates for patients with CKD are higher than for those without CKD. End stage renal disease (ESRD) patients on dialysis also have a high mortality and ESRD patients use a disproportionately high burden of any healthcare budget. Medicare spending for patients with CKD ages 65 and older exceeded $\$ 50$ billion in 2014 and represented 20 percent of all Medicare spending in this age group. ${ }^{6}$ Thus, intervention before this stage can be cost effective and increase life expectancy.

Telemedicine or tele-nephrology provides coordinated and collaborative care to improve patient outcomes. As the CKD burden increases, tele-nephrology is a viable option to facilitate communication between primary care physicians and nephrologists, and patients, ensuring optimal care. Tele-nephrology can reduce referral time, response time, and cost, and has been adopted in European countries. ${ }^{7}$ Tele-nephrology has been shown to stabilize GFR and improve blood pressure control in CKD patients in a study conducted in the Miami VA Healthcare System. ${ }^{8}$

In addition, telemedicine/tele-nephrology can be useful for those patients residing in remote areas. This has been demonstrated by a study from National Institute of Health. 
In this program, there was close collaboration between the nephrologist, nurses, pharmacist and the primary care provider. ${ }^{9}$

Our study demonstrates that closer monitoring of chronic kidney disease patients with the use of electronic medical records and the Internet is viable. Satisfaction rate among patients was high and clinical outcomes were significantly better than in those patients not monitored closely.

This study is limited by small sample size and short follow up. In addition, cost effectiveness was not studied. This same study design is now going to be used for a larger sample using remote monitoring devices, integrating automatically with the patients' electronic medical record, and the use of automatic feed back to the patients. This closed loop should ensure efficient, cost effective, coordinated and real time delivery of medical care.

Further research is needed to optimize tele-nephrology applications and subsequently improve patient outcomes. Impact of tele-nephrology on quality of life, patient time and cost saving must be determined. Responsibility and liability questions must be addressed, as well as ethical issues including privacy and confidentiality. In addition, adherence to established clinical guidelines must be ensured. ${ }^{10}$

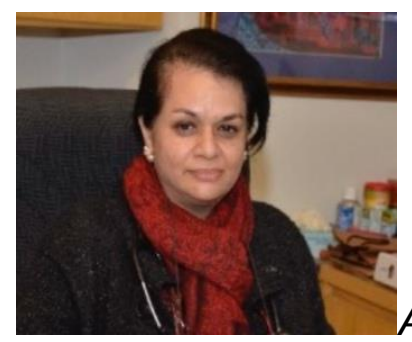

Abeera Mansur, MBBS, FACP, is Diplomate American Board of Internal Medicine and Nephrology (USA) in 1997, her special interests include Kidney diseases, Hypertension and Hemodialysis. She graduated in 1988 and is presently working as a Consultant Nephrologist <abeeramansur@hotmail.com>. 


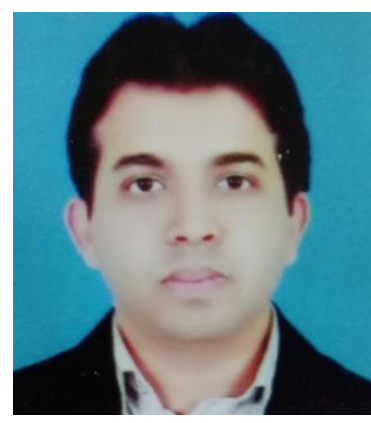

Rehan Javed, MBBS, Member ERA-EDTA, is currently working in nephrology and coordinates the tele-nephrology program.

\section{References}

1. United States Renal Data System. Chapter 1: CKD in the General Population. 2015 USRDS annual data report: Epidemiology of Kidney Disease in the United States. Bethesda, MD: National Institutes of Health, National Institute of Diabetes and Digestive and Kidney Diseases; 2015.

2. Centers for Disease Control and Prevention. Prevalence of chronic kidney disease and associated risk factors--United States, 1999-2004. MMWR. 2007 Mar 2. 56(8):161-5.

3. Coresh J, Selvin E, Stevens LA, et al. Prevalence of chronic kidney disease in the United States. JAMA. 2007;298(17):2038-47.

4. Long-term trends in the prevalence of chronic kidney disease in the United States.

Centers for Disease Control and Prevention (CDC)—Chronic Kidney Disease Surveillance-2017.

5. Hoerger TJ, Simpson SA, Yarnoff BO, et al. The future burden of CKD in the United States: a simulation model for the CDC CKD Initiative. Am J Kidney Dis. 2015;65(3):403-11

6. United States Renal Data System. Chapter 6: Medicare Expenditures for Persons with CKD. 2016. URL: https://www.usrds.org/2016/view/v1 06.aspx. Accessed $5 / 24 / 17$. 
7. Nynke D. Scherpbier-de Haan, MD, et al. Initial Implementation of a web-based consultation process for patients with chronic kidney disease.. Ann Fam Med.2013 Mar; 11(2):151-6.

8. Ladino MA, Wiley J, Schulman IH, et al. Tele-Nephrology: A Feasible Way to Improve Access to Care for Patients with Kidney Disease Who Reside in Underserved Areas. Telemed J E Health. 2016 Aug;22(8):650-4.

9. Narva AS1, Romancito G2, Faber T2, Steele ME2, Kempner KM2. Managing CKD by Telemedicine: The Zuni Tele-nephrology Clinic. Adv Chronic Kidney Dis. 2017 Jan;24(1):6-11.

10. Schoenfeld AJ, Davies JM, Marafino BJ, et al. Variation in Quality of Urgent Health Care Provided During Commercial Virtual Visits. JAMA Intern Med. 2016 May $1 ; 176(5): 635-42$.

Category: Original research

Tags: chronic kidney disease, cost effective, management, tele-nephrology, telehealth, telemedicine 